\title{
Can Whole Slide Imaging Replace Conventional Microscopic Evaluation? A Comparative Study over a Spectrum of Cases
}

\author{
Rajeswari Jayakumar, MD'; Nicholas Cassai²; Derek Laskar, MD; \\ Nigar Anjuman Khurram, MD'; Charles LaDoulis, MD²; Rosemary Wieczorek, MD ${ }^{1,2}$; \\ Matthew R. Pincus, MD, PhD ${ }^{1 *}$
}

\author{
Department of Pathology, SUNY Downstate Medical Center, Brooklyn, NY, USA \\ 2 Pathology and Laboratory Medicine Service, VA New York Harbor Health Care System, Brooklyn, NY, USA
}

\begin{abstract}
Whole slide scanning has made digital pathology and telepathology a reality. The quality of the image is crucial to enable accurate diagnosis. The purpose of this pilot study was to compare the agreement between diagnosis by scanned slides and conventional microscopy. Fifty-eight surgical pathology cases with a variety of diagnosis from different organs were retrieved. Single slides from each of these cases were scanned, and both the scanned slides and the original glass slides were reviewed at low and high power by two independent pathologists using both conventional microscopy and scanned slides. At low power, for most of the slide pairs, it was more difficult to evaluate the scanned slides because most of these slides had less well-defined architectural features. Nonetheless, there was 100 percent agreement in diagnosis for each pair of slides for all 58 cases. At high power, it was significantly more difficult to evaluate the scanned slides because of loss of cytologic detail on the scanned slide that was visible on the original glass slide. However, since, in some of the cases with low cellularity and less complex cytology, the high-power images were of the same quality as on the original slide, the system seems to be amenable to improvement.
\end{abstract}

\section{Introduction}

Digital Pathology as a field has grown significantly in the last thirty years [1]. In recent years it is being utilized for various purposes including intra-operative consultation, quality assurance and teaching. Whole slide scanning can be an effective tool for telepathology $[2,3]$. The quality of the images needs to be carefully assessed to ensure that the accuracy of the diagnosis is not compromised. It is equally important to ascertain if cellular details are as clear as under microscopy in higher magnifications. This pilot study compares the agreement in diagnosis between scanned slides and conventional microscopy in both low and high magnifications.

\section{Materials and Methods}

Single slides from fifty-eight (58) surgical pathology cases were scanned using a Mikroscan D2-V2 Ver 1.1.130.19 whole slide scanner system which then provides televised projections of these slides. Each scanned slide and then its corresponding original glass slide were reviewed at low and high power by two pathologists independently first using the televised projections of each scanned slide and then conventional microscopy on each corresponding original glass slide. The cases included 15 malignancies, 19 benign neoplasms (mainly colonic adenomas or hyperplasias), 3 normal tissues including a bone marrow biopsy, 5 inflammatory states, 10 benign dermatopathological cases and 6 miscellaneous cases (including 1 calcified valve leaflet, 1 atherosclerotic plaque, 1 steatohepatitis, 1 gastropathy, 1 colonic mucosa with lymphoid aggregates and 1 cataract). The quality of the scanned slide in comparison to conventional microscopy was semi-quantitatively scored, using a scoring system from 0 to 4 , with score 0 being poor quality of the scanned slide and score 4 being excellent quality. The scoring was performed at both low and high magnification. The highest achievable score was 232, i.e., grade of 4 on all 58 cases.

\section{Results}

The highest score for any individual case was 3 (very good). Diagnosis of all 58 cases was identical by both scanned slide and conventional micros- copy by both pathologists ( $100 \%$ agreement) at both low and high power. The overall scores given for the cases at low and high power by each pathologist is given in Table 1.

As can be seen in this table, both pathologists evaluated the scanned slides as being of significantly lower quality than the original glass slides. These are reflected in the overall scores for the low scanned slides where there was close agreement between the two scores, 110 and 113, both of which are significantly less than the highest score of 232.

The scores for the high-power slides were even lower compared with the best possible score of 232 (Table 1). Although these scores differed between the two pathologists, 58 and 87 , both scores were much lower than those for the corresponding low power comparisons. This was caused by the absence of morphologic details on the high power scanned slides. While it was possible to make the correct diagnosis on all 58 scanned high-power slides, these slides would be inadequate for providing critical information that is often required. For example, Modified Richardson Scoring for breast cancer, assessing mitotic count, identifying single cell necrosis, identifying lymphovascular or perineural invasion, Fuhrman scoring of renal neoplasms etc. which needed crisp cellular details at high power were not assessable with accuracy using scanned slides compared to conventional microscopy resulting in the lower scores for the scanned high-power slides. In contrast, definitive diagnoses were

Table 1. The Overall Scores Given by Each Pathologist at Low and High Power for the 58 Cases, Each Case Being Scored on a Scale of 0 (Poor Quality of Scanned Slide) to 4 (Excellent Quality of Scanned Slide)

\begin{tabular}{|l|c|c|}
\hline Pathologist & Low power score & High power score \\
\hline Pathologist 1 & 110 & 58 \\
\hline Pathologist 2 & 113 & 87 \\
\hline
\end{tabular}

*Scores reported are the cumulative semi quantitative score of all 58 cases. 
made much more easily by examining overall architectural features of the scanned slide under low power resulting in higher score at lower magnification.

The pathologists encountered difficulty especially with cases with high cellularity or cases with subtle differences between individual cells such as bone marrow biopsy. Certain cases with monotonous and bland cytology such as low grade neuroendocrine tumors, malignant bone marrow specimens and dermatologic malignancies had good correlation between conventional microscopy and scanned slides at high power. Also, the pathologists observed that the slide section thickness did affect the quality of the scanned images. Slides with thinner sections produced better images than the ones with thicker sections.

\section{Discussion}

Digital pathology has gained huge momentum in this decade with new developments each year. It has several advantages including cost savings, improved performance and work-flow. It is also a great tool for telepathology [4]. Recent advances in computer-based diagnostics have made whole slide scanning appear to be replacing conventional microscopy in coming years. Despite these advantages, the process of convincing practicing pathologists to adapt to digital images and to shift them from conventional microscopy is a great challenge. The quality of the digital images is one of the key factors that would influence pathologists to accept or reject this modality of slide review. Although the performance of most scanners is commendable at low magnification, the same does not hold true in higher magnifications. It is essential to address this problem as pathologic diagnosis of most cases are arrived at based on the architecture of the tissue at low magnification along with the cytologic details appreciated at higher magnification. Hence the quality of the image cannot be compromised on either magnification.

In our study, we graded the quality of images with ' 0 ' being poor quality and ' 4 ' being excellent quality. As we had 58 cases in total, the highest achievable score was 232. The two pathologists who reviewed the slides gave higher scores for these cases at lower magnification than higher magnification. The relatively low scores of 58 and 87 at higher magnification indicate that the system is not ready yet to be employed as an alternative to conventional microscopy. For example, prostate adenocarcinomas of Gleason score 4 and above are easy to identify with the scanned images while Gleason score 3 and details including perineural and lymphovascular invasion were not detected on the scanned slides. Although there was hundred percent agreement with the diagnosis between microscopy and scanned images, it is important to emphasize that diagnosis alone is not sufficient in most cases as there are other details that need to be documented in the pathology report that are of therapeutic and prognostic significance. These include such observations as nuclear grade, mitotic counts, lymphovascular invasion and capsular invasion to name a few.

The pathologists primarily relied on low power images to arrive at a diagnosis in our study. We ensured to include a wide range of diagnosis in our study from normal to benign to malignant tumors to avoid any possible bias due to complex architecture and cytologic features specific to a particular diagnosis. Although the low power scores were higher, it still did not seem to be quite satisfactory as the scores were nearly half (110 and 113) of what was highest possible score (232). The highest score for any individual case was 3. None of the images were of excellent quality (grade 4) on both magnifications.

Whole slide scanners should also be able to reproduce the image consistently after multiple scans. It is a known problem that the same slide scanned by same scanner at different times may appear different due to external factors including temperature and mechanical shifts. With the advent of computer-based diagnostics, high quality and reproducibility of scanned images can directly impact the diagnosis [5]. Quality of the image is also affected by factors such as thickness of the section, staining and fixation which are difficult to standardize. Studies show that thickness of the section affects the color appearance and details of the image with thinner sections demonstrating clearer details and thicker sections showing unclear details which was also the case with our study [6].

Multiple studies have shown good concordance between the diagnosis by conventional microscopy and whole slide images. Studies comparing diagnostic accuracy and agreement between glass and virtual slides by Mooney et al. on dermatopathology cases, Chargari et al. and Fine et al. on prostate needle core biopsies, Gilbertson et al. on genitourinary and dermatopathology cases all reiterated that there is excellent concordance between the two methods [7-10]. Studies by Tsuchihashi et al. and Fallon et al. revealed that whole slide scanned images can be helpful for frozen section diagnosis where the frozen artifacts sometimes make it difficult to interpret even by conventional microscopy $[11,12]$.

\section{Conclusion}

Our study showed 100 percent concordance between conventional microscopy and scanned images for the diagnosis alone while further delineation of grading or assessment of more details at the cellular level was not convincing enough by scanned slides to replace conventional microscopy. However, with the current configurations, this system may be adequate for routine histopathological diagnosis which does not necessarily need high power for complete diagnosis. This system seems to be amenable to improvement as there were several cases with clear cellular morphology at higher magnification in our study.

\section{Article Information}

*Correspondence: Matthew R. Pincus, MD, PhD

Professor, Department of Pathology, SUNY Downstate Medical Center, 450 Clarkson Avenue, Brooklyn, NY, USA. Email: matthew.pincus@downstate.edu

Received: Dec. 20, 2017; Accepted: Mar. 5, 2018; Published: Apr. 30, 2018

\section{DOI: 10.24983/scitemed.jacp.2018.00060}

Copyright (c) 2018 The Author (s). This is an open-access article distributed under the terms of the Creative Commons Attribution 4.0 International License (CC-BY).

Disclosure: A preliminary version of this study was presented in poster form at the $2^{\text {nd }}$ Digital Pathology Congress USA, July 14-15, 2016, Philadelphia, PA, USA.

\section{Funding: None}

\section{Conflict of Interest: None}

\section{Keywords}

Conventional microscopy; grading system; morphological features; perineural and lymphovascular invasion; whole slide imaging.

\section{References}

1. Weinstein RS, Bloom KJ, Rozek LS. Telepathology and the networking of pathology diagnostic services. Archives of Pathology \& Laboratory Medicine 1987;111(7):646-652.

2. Dee FR, Meyerholz DK. Teaching medical pathology in the twenty-first century: Virtual microscopy applications. Journal of Veterinary Medical Education 2007;34(4):431-436.

3. Vitkovski T, Bhuiya T, Esposito M. Utility of telepathology as a consultation tool between an off-site surgical pathology suite and affiliated hospitals in the frozen section diagnosis of lung neoplasms. Journal of Pathology Informatics 2015;6:55.

4. Ghaznavi F, Evans A, Madabhushi A, Feldman M. Digital imaging in 


\section{ORIGINAL}

pathology: Whole-slide imaging and beyond. Annual Review of Pathology: Mechanisms of Disease 2013;8:331-359.

5. Shrestha P, Kneepkens R, Vrijnsen J, Vossen D, Abels E, Hulsken B. A quantitative approach to evaluate image quality of whole slide imaging scanners. Journal of Pathology Informatics 2016;7:56.

6. Yagi Y. Color standardization and optimization in whole slide imaging. Diagnostic Pathology 2011;6(Suppl 1):S15.

7. Mooney E, Hood AF, Lampros J, Kempf W, Jemec GB. Comparative diagnostic accuracy in virtual dermatopathology. Skin Research and Technology 2011;17(2):251-255.

8. Chargari C, Comperat E, Magne N, et al. Prostate needle biopsy examination by means of virtual microscopy. Pathology, Research and Practice 2011;207(6):366-369.
9. Fine JL, Grzybicki DM, Silowash R, et al. Evaluation of whole slide image immunohistochemistry interpretation in challenging prostate needle biopsies. Human Pathology 2008;39(4):564-572.

10. Gilbertson JR, Ho J, Anthony L, Jukic DM, Yagi Y, Parwani AV. Primary histologic diagnosis using automated whole slide imaging: a validation study. BMC Clinical Pathology 2006;6:4.

11. Tsuchihashi $Y$, Takamatsu $T$, Hashimoto $Y$, Takashima $T$, Nakano K, Fujita S. Use of virtual slide system for quick frozen intra-operative telepathology diagnosis in Kyoto, Japan. Diagnostic Pathology 2008;3(Suppl. 1):S6.

12. Fallon MA, Wilbur DC, Prasad M. Ovarian frozen section diagnosis: use of whole-slide imaging shows excellent correlation between virtual slide and original interpretations in a large series of cases. Archives of Pathology \& Laboratory Medicine 2010;134(7):1020-1023. 